\title{
Bedside Assessment of the Necessity of Daily Lab Testing for Patients Nearing Discharge
}

\author{
Surafel Tsega, MD*, Michelle O'Connor², Jashvant Poeran, MD, PhD'1,3, Colin lberti, MD', Hyung J. Cho, MD
}

'Department of Medicine, Mount Sinai Hospital, New York, New York; ${ }^{2}$ lcahn School of Medicine at Mount Sinai, New York, New York; ${ }^{3 / n s t i t u t e ~ f o r ~}$ Healthcare Delivery Science, Department of Population Health Science \& Policy, New York, New York.

As part of the Choosing Wisely ${ }^{\circledR}$ campaign, the Society of Hospital Medicine recommends against performing "repetitive complete blood count chemistry testing in the face of clinical and lab stability." With this recommendation as a framework, we targeted 2 hospitalist-run inpatient medicine units that employed bedside, scripted, interdisciplinary rounds. Our multifaceted intervention included prompting the hospitalist to identify clinically stable patients for next-day discharge and to discontinue labs when appropriate. It was coupled with the education of the clinicians and a regular data review for the hospitalists and unit staff. Among 2877 discharges included in a 1-year period, there was a significantly decreasing trend after the intervention in the percentage of patients getting labs in the 24,48 , and 72 hours before discharge $(-1.87 \%,-1.47 \%$, and $-0.74 \%$ decrease per month, respectively; $P<.05)$. Our structured, multifaceted approach effectively reduced daily lab testing in the 24 to 48 hours prior to discharge. Journal of Hospital Medicine 2018;13:38-40. Published online first October 18, 2017. (C) 2018 Society of Hospital Medicine

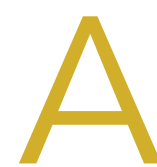

s part of the Choosing Wisely ${ }^{\circledR}$ campaign, the Society of Hospital Medicine recommends against performing "repetitive complete blood count $[\mathrm{CBC}]$ and chemistry testing in the face of clinical and lab stability." 1 This recommendation stems from a body of research that shows that frequent or excessive phlebotomy can have negative consequences, including iatrogenic anemia (termed hospital-acquired anemia), which may necessitate blood transfusion. ${ }^{2}$ The downstream effects of potentially unnecessary testing, including the evaluation of false-positive results, must also be considered. Additional important effects include patient discomfort and disruption of sleep and unproductive work by hospital staff, including nurses, phlebotomists, and laboratory technicians.

Though interventions to reduce unnecessary daily labs have been previously evaluated, there are no studies that focus on decreasing lab testing on patients deemed clinically stable and close to discharge. This is in part due to the absence of clear criteria or guidelines to define clinical stability in the context of lab utilization.

We therefore aimed to implement a multifaceted, patient-centered initiative-the Necessity of Labs Assessed Bedside (NO LABS) - that focused on reducing lab testing in patients at 24 to 48 hours before discharge. We targeted the 24 to 48 -hour period before the anticipated date of discharge, as

*Address for correspondence and reprint requests: Surafel Tsega, MD, Mount Sinai Health System, One Gustave L Levy Place, Box 1086, New York, NY 10029; Telephone: 212-241-1653; Fax: 212-289-6393; E-mail: surafel.tsega@mountsinai. org

Received: May 12, 2017; Revised: July 17, 2017; Accepted: July 20, 2017

2018 Society of Hospital Medicine DOI 10.12788/jhm.2869 this may be a period of greater stability and provide an opportunity to identify and decrease unnecessary testing.

\section{METHODS}

The study took place at Mount Sinai Hospital, which is an 1174bed tertiary care teaching hospital in New York City. We targeted 2 inpatient medicine units where virtually all patients are assigned to a hospitalist rotating for a 2- to 4-week period, for the period of July 1, 2015, to July 31, 2016. These units employed bedside interdisciplinary rounds (IDR) attended by the hospitalist, social worker, case manager, nurse, nurse manager, and medical director. Bedside IDR focuses on the daily plan and patient safety by utilizing a scripted format. ${ }^{3}$ Our multifaceted intervention included prompting the hospitalist physician during bedside IDR, education of the clinicians, and regular data review for the hospitalists and unit staff.

As described by Dunn et al., ${ }^{3}$ the IDR script included the following: a review of the plan of care by the hospitalist, identifying a patient's personal goals for the day, a brief update of discharge planning (as appropriate), and a safety assessment performed by the nurse (identifying Foley catheters, falls risk, etc). We incorporated an inquiry into the daily IDR script identifying clinically stable patients for discharge in the next 24 to 48 hours (based on physician judgment), followed by a prompt to the hospitalist to discontinue labs when appropriate. The unit medical director and nurse manager were both tasked with prompting the hospitalist at the bedside. Our hospital utilizes computerized physician order entry. Lab orders were then discontinued by the clinician during rounds using a computer on wheels (or after rounds when one was not available). The hospitalist, unit medical director, and nurse manager were reminded about the project through weekly e-mails and in-person communication. 


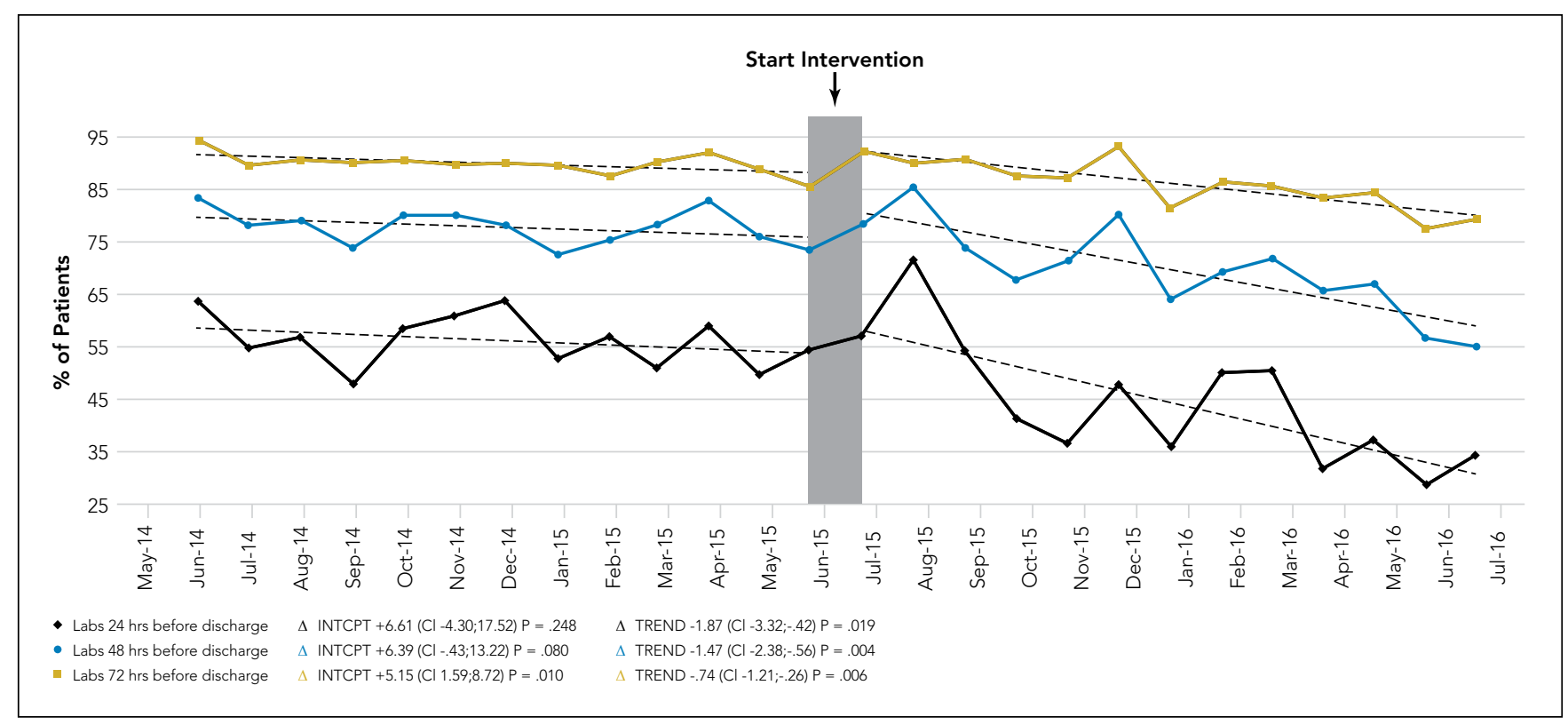

FIG. Monthly percentage of patients with labs ordered in days prior to discharge.

NOTE: Interrupted time-series analysis results showing monthly percentage of patients with labs ordered in the $24 / 48 / 72$ hours preceding discharge. $\triangle I N T C P T$ and $\triangle T R E N D$ depict the change in intercept and slope, respectively, between the pre and postintervention periods. The gray bar identifies the start of the intervention period.

To assess whether the prompt was being incorporated consistently, an observer was added to rounds beginning in the second month of the project. The observer was present at least 3 times a week for the subsequent 3 months of the project. Our intervention also included education geared towards hospitalists, including a brief presentation on reducing unnecessary lab testing during a monthly hospitalist faculty meeting (the first and sixth month of the intervention). The group's data on laboratory testing within the 24 to 48 hours prior to discharge were also presented at these monthly meetings (beginning 2 months into the intervention and monthly thereafter). Lastly, we provided the unit staff with unit-level metrics, biweekly for the first 3 months and every 2 to 3 months thereafter.

We extracted electronic medical record (EMR) data on lab utilization for patients on the 2 hospitalist units for the intervention period. Baseline data were obtained from July 1, 2014, to June 30,2015 . Patients with a length of stay (LOS) $\leq 7$ days (75th percentile) were included; on these units, longer stays were considered more likely to have complex social issues delaying discharge and thus less likely to require laboratory testing. We tracked ordering for 4 common lab tests: basic metabolic panel, CBC, CBC with differential, and the comprehensive metabolic panel. The primary outcome was the monthly percentage of patients for whom testing was ordered in the 24 and 48 hours preceding discharge. A secondary outcome was testing at 72 hours preceding discharge to identify any potential compensatory (increased) testing the evening prior. We applied a quasi-experimental interrupted time series design with a segmented regression analysis to estimate changes before and after our intervention, expressed in acute changes (change in intercept) and over time (changes in trend) while adjusting for preintervention trends. All analyses were performed with SAS v9.4 statistical software (SAS Institute, Cary, NC). Our project was deemed a quality improvement project, and thus an IRB submission was not required.

\section{RESULTS}

There were 1579 discharges in the preintervention period and 1308 discharges in the postintervention period. The average age of the patient population was similar in the baseline and postintervention groups (61.5 vs 59.3 years; $P=.400$ ), and there was no difference in the mean LOS before and after implementation (3.67 vs 3.68 days; $P=.817$ ).

There was a significant decrease in the average percentage of patients with any lab order at 24 hours prior to discharge, from a preintervention average of $50.1 \%$ to a postintervention average of $34.5 \%(P=.004)$. Similarly, labs ordered at 48 hours prior to discharge also decreased (from $77.6 \%$ down to $55.1 \%$; $P=.005)$. This corresponded to a significantly decreasing trend (relative to the preintervention period) in the percentage of patients getting labs after the intervention in the 24,48 , and 72 hours before discharge $(-1.87 \%[P=.019],-1.47 \%[P=.004]$, and $-0.74 \%[P=.006]$ decrease per month, respectively; Figure). There was an initial period of increased lab testing at 72 hours before discharge $(+5.15 \%$; $P=.010)$; however, by the fifth month of the project, testing reached preintervention levels and was followed by a sustained decrease in testing. When assessing the entire hospitalization, we saw a decrease in the mean number of labs ordered per patient day, from 1.96 down to 1.83 post intervention $(P=.0101)$.

\section{DISCUSSION}

Our structured, multifaceted approach effectively reduced daily lab testing in the 24 to 48 hours prior to discharge. Bedside IDR provided a unique opportunity to effectively communicate to the patient about necessary (or unnecessary) testing. More- 
over, given the complexity of identifying clinical stability, our strategy focused on the onset of discharge planning, a more easily discernible and less obtrusive focal point to promote the discontinuation of lab testing.

Though the nature of bundled interventions can make it difficult to identify which intervention is most effective, we believe that all interventions were effective in different capacities during various phases in the intervention period. We believe that the decrease in lab testing in the 24 to 48 hours preceding discharge was primarily driven by the new rounding structure. This is evident in the significant decrease seen in the first few months of the intervention period. Six months into the intervention, we begin to see a decrease at 72 hours prior to discharge. Additionally, we see a decrease in the mean number of labs per patient day over the entire hospitalization period. We attribute these results to a gradual shift in the culture in our division as a direct consequence of educational sessions and individual feedback provided during this time.

To our knowledge, this is the first study to use anticipated discharge as a correlate for clinical stability and therefore as an opportunity to prompt discontinuation of laboratory testing. Other studies evaluated interventions targeting the EMR and the ease with which providers can order recurring labs. These include restricting recurring orders in the $\mathrm{EMR}^{4}{ }^{4}$ a robust education and awareness campaign targeting house staff, ${ }^{5}$ and other multifaceted approaches to decreasing lab utilization, ${ }^{6}$ all of which have shown promising results. While these approaches show varying degrees of success, ours is unique in its focus on the period prior to discharge. In addition, the intervention can

\section{References}

1. Bulger J, Nickel W, Messler J, et al. Choosing wisely in adult hospita medicine: Five opportunities for improved healthcare value. J Hosp Med. 2013;8(9):486-492.

2. Thavendiranathan P, Bagai A, Ebidia A, Detsky AS, Choudhry NK. Do blood tests cause anemia in hospitalized patients? The effect of diagnostic phlebotomy on hemoglobin and hematocrit levels. J Gen Intern Med. 2005:20(6):520-524.

3. Dunn AS, Reyna, M, Radbill B, et al. The impact of bedside interdisciplinary rounds on length of stay and complications. J Hosp Med. 2017;3:137-142.

4. Iturrate E, Jubelt L, Volpicelli F, Hochman K. Optimize Your Electronic Medical Record to Increase Value: Reducing Laboratory Overutilization. Am J Med. 2016;129(2):215-220.

5. Wheeler D, Marcus P, Nguyen J, et al. Evaluation of a Resident-Led Project to be readily implemented in settings that utilize scripted IDR. It also brings high-value decision-making to the bedside by informing the patient that in the setting of presumed clinical stability, no additional tests are warranted.

Our study has several limitations. First, while interdisciplinary discharge rounds are widely implemented, 7,8 our rounds occur at the bedside and employ a script, potentially limiting generalizability. The structured prompting may be feasible during structured IDR in a standard conference room setting, though we did not assess this model. Second, bedside rounds only included patients who were able to participate. Rounding on patients unable to participate, such as patients with delirium with agitation, was done outside the patient room rather than at the bedside. A modified script was used in these instances (absent questions addressed to the patient), allowing for the prompt to be incorporated. These patients were included in the analysis. Lastly, as previously stated, we cannot clearly identify which intervention (the prompt, education, or feedback) most effectively led to a sustained decrease in lab ordering.

Our structured, multifaceted intervention reduced laboratory testing during the last 48 hours of admission. Hospitals that aim to decrease potentially unnecessary lab testing should consider implementing a bundle, including a prompt at a uniform and structured point during the hospitalization of patients who are expected to be discharged within 24 to 48 hours, clinician education, an audit, and feedback.

Disclosure: All authors have no conflicts of interest to disclose.

Decrease Phlebotomy Rates in the Hospital: Think Twice, Stick Once. JAMA Intern Med. 2016;176(5):708-710.

6. Corson AH, Fan VS, White T, et al. A Multifaceted Hospitalist Quality Improvement Intervention: Decreased Frequency of Common Labs. J Hosp Med. 2015; 10(6):390-395

7. Bhamidipati VS, Elliott DJ, Justice EM, Belleh E, Sonnad SS, Robinson EJ. Structure and outcomes of interdisciplinary rounds in hospitalized medicine patients: A systematic review and suggested taxonomy. J Hosp Med. 2016;11(7):513-523.

8. O'Leary, KJ, Sehgal NL, Terrell G, Williams MV, High Performance Teams and the Hospital of the Future Project Team. Interdisciplinary teamwork in hospitals: a review and practical recommendations for improvement. $J$ Hosp Med. $2012 \cdot 7(1): 48-54$ 